\title{
Relativistic satellite astrometry: the stellar radial velocity
}

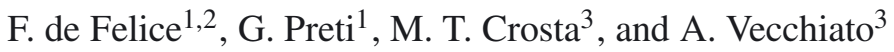

\author{
1 Dipartimento di Fisica “Galileo Galilei”, Università degli Studi di Padova, via Marzolo 8, 35131 Padova, Italy \\ e-mail: [defelice;preti]@pd.infn.it, [crosta;vecchiato]@to.astro.it \\ 2 INFN, Sezione di Padova, via Marzolo 8, 35131 Padova, Italy \\ 3 Osservatorio Astronomico di Torino, strada Osservatorio 20, 10125 Pino Torinese (TO), Italy
}

Received 12 July 2010 / Accepted 6 January 2011

\begin{abstract}
We present a general-relativistic analysis that allows the stellar radial velocities to be determined from a suitable implementation of the spectroscopic data with the astrometric ones shortly to be provided by new generations of astrometric satellites at $\mu$ arcsec levels of accuracy at least. This analysis leads to an enhancement of the $c^{-1}$ Doppler-shift formula presently planned for the Gaia mission to an all-inclusive, general-relativistic formula at the $c^{-3}$ level, consistently with the expected accuracy. From this formula, which is shown to provide relevant corrections already at the $c^{-2}$ level to a previously proposed one, we are then able to derive the explicit expression for the stellar radial velocity in terms of the spectroscopic and astrometric data, thereby accounting for all the necessary relativistic corrections up to and including the $c^{-3}$ level.
\end{abstract}

Key words. gravitation - methods: analytical - techniques: high angular resolution - techniques: spectroscopic - astrometry - stars: kinematics and dynamic

\section{Introduction}

The new generation of astrometric satellites - like Gaia, which is going to be launched by ESA within the next few years - will be able to provide astronomers with a wealth of information about the structure of the Galaxy, thanks to the impressive accuracy in the measurement capabilities (Turon et al. 2005). For instance, Gaia is expected to reach $\mu$ arcsec precision in measuring the angular distances, thus outperforming the presently employed Hipparcos data by a factor of $10^{3}$ and thereby providing us with an accurate description of the star positions up to the boundary of the Galaxy, some $10 \mathrm{kpc}$ away.

Such a high accuracy in the expected astrometric performance implies that the general-relativistic effects must be taken into account in analysing the experimental data provided by these satellites, since the $c^{-3}$ level of precision is mandatory for this analysis to be consistent with the $\mu$ arcsec accuracy of their astronomical observations. To this end, a "Relativistic Astrometric MODel" (RAMOD) has been conceived and developed - see de Felice et al. (1998), de Felice et al. (2001), Bini $\&$ de Felice (2003), Bini et al. (2003), de Felice et al. (2004), de Felice et al. (2006), de Felice \& Preti (2006), de Felice \& Preti (2008), Crosta \& Vecchiato (2010), and Preti \& de Felice (2010) - providing a fully general-relativistic analysis of the inverse ray-tracing problem at the required $c^{-3}$ level from the observational data (the direction cosines of the detector's line-ofsight) back to the position of the light-emitting star. Resting on this theoretical framework, we derive here an explicit formula for determining the stellar radial velocity, taking advantage of both the spectroscopic and the astrometric data supplied by the observations.

As a matter of fact, this new generation of astrometric satellites will not only allow us to greatly enhance our knowledge of the Galactic structure as far as the stellar positions are concerned, but also provide precise information allowing astronomers to frame a much more detailed kinematical picture of our Galaxy than the presently available one. To this end, these satellites will also be equipped with high-precision spectrometers, allowing determination of the stellar radial velocities on the basis of the Doppler shift of the stellar spectral lines. The interpretation of these observational data in terms of radial velocities is by no means a trivial task yet (Gullberg \& Lindegren 2002; Lindegren \& Dravins 2003). Moreover, as we note in Sect. 2, the Doppler-shift formula that is planned for the spectroscopic data analysis (de Bruijne et al. 2005) in the case of the first of these new-generation satellites, Gaia, does not take advantage of the high accuracy of its expected astrometric performance at all. After recalling in Sect. 3 the general-relativistic astrometric framework in which we operate, we thus introduce in Sect. 4 an entirely new method that allows implementing the spectroscopic data with the astrometric ones, thus leading to determining the exact general-relativistic Doppler-shift formula at the required $c^{-3}$ level. In Sect. 5, this formula will be compared to a previously proposed one (Lindegren \& Dravins 2003), and this comparison will reveal how our consistently general-relativistic analysis will correct the latter. The issue of separating the radial velocity contribution from that of the proper motion is discussed in Sect. 6. In Sect. 7 an explicit solution of the $c^{-3}$ equation for the radial velocity is obtained, and in Sect. 8 we finally draw the conclusions of our paper.

As notation and conventions, the metric signature +2 is employed. Greek (spacetime) indices run from 0 to 3, while Latin (space) indices run from 1 to 3 . From now on, the expression $O(n)$ is used to indicate terms of the order of $c^{-n}$; moreover, in all the formulae the $G$ and $c$ factors will be set equal to 1 .

\section{The Doppler-shift formula}

Comparison of the observed spectrum of a star with a reference one allows the stellar radial velocity to be determined via 
the cross-correlation method, based on measurement of the frequency shift between the pairs $\left(\omega_{*}, \omega_{\text {obs }}\right)$ of corresponding frequencies, where $\omega_{*}$ is the frequency of a photon as emitted by the star, and $\omega_{\text {obs }}$ the corresponding ("cross-correlated") frequency measured by the satellite rest observer.

In the case of Gaia and according to the official specifications for its spectroscopic performance (de Bruijne et al. 2005), the stellar radial velocity $v_{*_{\text {rad }}}$ will be determined as

$v_{*_{\mathrm{rad}}}=\frac{\omega_{*}}{\omega_{\mathrm{obs}}}-1$,

hence employing a Doppler-shift formula that does not take any relativistic corrections at all into account, even if they are primary for the expected astrometric observations. These corrections, however, cannot be provided simply by the well-known special-relativistic formula

$\frac{\omega_{\mathrm{obs}}}{\omega_{*}}=\frac{1-v_{*_{\mathrm{rad}}}}{\sqrt{1-v_{*}^{2}}}$,

because the genuinely general-relativistic regime in which the future astrometric missions will operate naturally calls for a fully general-relativistic Doppler-shift formula, including all the corrections due to the gravitoelectric and gravitomagnetic effects that a dynamical curved spacetime background has on the motion of the photon from the star to the satellite. The simple local comparison of $\omega_{*}$ with the corresponding $\omega_{\text {obs }}$, as implied by Eq. (2), does not consider the whole story of the light ray from its origin to its observation, which causes relevant terms to be neglected in the determination of the stellar velocities. As we see, implementing the Doppler-shift data with the information coming from the astrometric observations allows all the necessary relativistic corrections to be naturally included in a generalrelativistic Doppler-shift formula that is fully consistent with the $O(3)$ accuracy, at least, of the new generation of astrometric satellites.

\section{The relativistic astrometric framework}

We have already recalled that the use of a general-relativistic environment is mandatory for consistent dealing with $\mu$ arcsecprecision data; accordingly, the behaviour of the light ray stemming from a distant star and reaching the satellite detector must be analysed in terms of geodesic propagation in a curved spacetime. The expression for the background metric in which the light ray propagates is written in the form

$\mathrm{d} s^{2}=g_{\alpha \beta} \mathrm{d} x^{\alpha} \mathrm{d} x^{\beta}=\left(\eta_{\alpha \beta}+h_{\alpha \beta}+O\left(h^{2}\right)\right) \mathrm{d} x^{\alpha} \mathrm{d} x^{\beta}$,

corresponding to the weak-field, slow-motion approximation; the sources of gravity are assumed to be the bodies of the solar system alone. In Eq. (3), the $h_{\alpha \beta}$ terms represent perturbations to the Minkowski metric $\eta_{\alpha \beta}$, and the expression $O\left(h^{2}\right)$ denotes nonlinear terms in these perturbations. The $h_{\alpha \beta}$ terms encapsulate the gravitoelectric and gravitomagnetic potentials of the gravitational sources, whose contributions we need to retain up to and including the $O(3)$ level, in order to comply with the accuracy of the astrometric measurements, as in the case of Gaia. To the required order of precision, we have

$h_{00} \sim O(2), \quad h_{0 i} \sim O(3), \quad h_{i j}=h_{00} \delta_{i j} \sim O(2)$,

and the metric components of Eq. (3) are given by

$$
\begin{aligned}
g_{00} & =-1+h_{00}+O(4), \\
g_{0 i} & =h_{0 i}+O(5), \\
g_{i j} & =\delta_{i j}+h_{i j}+O(4) .
\end{aligned}
$$

Following the IAU resolution B1.3 (2000), we introduce a family of locally baricentric observers $\mathbf{u}$ who fill the spacetime (cf. de Felice et al. 2006), each of them at rest with respect to the Barycentric Celestial Reference System (BCRS). Explicitly, we have

$u^{\alpha}=\frac{\delta_{0}^{\alpha}}{\sqrt{-g_{00}}}$

where $g_{00}$ is evaluated at the specific spacetime point where the particular observer $\boldsymbol{u}$ belonging to this family is located. With respect to $\boldsymbol{u}$, we can define a spacelike vector $\boldsymbol{\ell}$ which represents $\boldsymbol{u}$ 's local line of sight. The explicit expression for $\boldsymbol{\ell}$ reads as

$\ell^{\alpha}=P(u)^{\alpha}{ }_{\beta} k^{\beta}=k^{\alpha}+u^{\alpha} u_{\beta} k^{\beta}$,

where $\boldsymbol{k}$ is the tangent vector to the null geodesic followed by the light ray,

$P(u)^{\alpha}{ }_{\beta}=\delta_{\beta}^{\alpha}+u^{\alpha} u_{\beta}$

is the spatial projector on the local rest space of $\boldsymbol{u}$, and relation

$\ell^{\alpha} u_{\alpha}=0$

obviously holds.

Using Eqs. (3)-(7) for the metric, and introducing the convenient rescaled quantity

$\bar{\ell}^{\alpha}=-\frac{\ell^{\alpha}}{u^{\rho} k_{\rho}}$

which satisfies

$\bar{\ell}^{\alpha} u_{\alpha}=0, \quad \bar{\ell}^{\alpha} \bar{\ell}_{\alpha}=1$,

we are able to obtain (see de Felice et al. 2006) the equations of motion describing, at the required $O(3)$, the behaviour of the local line of sight along the light-ray path connecting the star with the observer. These "RAMOD master equations" explicitly read as

$$
\begin{aligned}
\frac{\mathrm{d} \bar{\ell}^{k}}{\mathrm{~d} \sigma}= & +\frac{1}{2} \bar{\ell}^{k} \bar{\ell}^{i} \bar{\ell}^{j} \partial_{0} h_{i j}-\bar{\ell}^{i} \bar{\ell}^{j} \delta^{k r}\left(\partial_{i} h_{r j}-\frac{1}{2} \partial_{r} h_{i j}\right) \\
& -\frac{1}{2} \bar{\ell}^{k} \bar{\ell}^{i} \partial_{i} h_{00}-\bar{\ell}^{i} \delta^{k r}\left(\partial_{i} h_{r 0}+\partial_{0} h_{r i}-\partial_{r} h_{0 i}\right) \\
& +\frac{1}{2} \delta^{k r} \partial_{r} h_{00}
\end{aligned}
$$

where $\sigma$ is a parameter suitably chosen along the null geodesic. The inverse ray-tracing algorithm based on Eq. (14) allows us to determine the stellar positions, the stellar motions, and the comprehensive error budget due to the uncertainties in the boundary conditions (de Felice \& Preti 2006 2008).

\section{The stellar velocity}

As shown in de Felice \& Preti (2006), all the components of the stellar velocities with respect to the BCRS can be determined directly from astrometric observations alone. However, when the astrometric satellites are also equipped with a high-precision spectrometer, as in the case of Gaia, a primary source of information will obviously come from the Doppler-shift data as well. We have seen above that the Doppler formula (1) that is actually going to be employed for Gaia's data analysis (de Bruijne et al. 2005) contains the Newtonian contribution alone; on the other hand, we have also seen that a $\mu$ arcsec astrometric performance 
implies that one has to consider the relativistic corrections up to and including the $O(3)$ level. As we now show, the relativistic astrometric model outlined in Sect. 3 is able to provide a Dopplershift formula that naturally includes all these corrections and allows determining the stellar radial velocity at this same $O(3)$.

The spatial three-velocity of a star relative to the local baricentric observer $\boldsymbol{u}$ located at the emission event is obtained from projecting the stellar four-velocity $\boldsymbol{u}_{*}$ on the rest space of this observer:

$v_{*}^{\alpha}=-\frac{P(u)^{\alpha}{ }_{\beta} u_{*}^{\beta}}{u_{*}^{\rho} u_{\rho}}=\frac{u_{*}^{\alpha}}{\gamma_{*}}-u^{\alpha}$

(cf. de Felice et al. 1990), where

$\gamma_{*}=-u_{*}^{\rho} u_{\rho}$

is the Lorentz factor of the star in the rest frame of $\boldsymbol{u}$. Recalling that the tangent four-vector to the light trajectory is $\boldsymbol{k}$, the emission frequency $\omega_{*}$ of a light signal is defined (cf. de Felice et al. 1990) as the frequency measured by the observer $\boldsymbol{u}_{*}$ comoving with the star, namely

$\omega_{*}=-\left(u_{*}^{\alpha} k_{\alpha}\right)_{*}$,

a quantity that is obviously evaluated at the star position in the asymptotic region of metric (3). The effect of the gravitational field of the emitting star can easily be taken into account via corrections to the background metric (3) at the star position as

$\left(g_{00}\right)_{*}=-1+\frac{2 M_{*}}{R_{*}}+O(4)$,

which can be evaluated using estimates for the mass $M_{*}$ and radius $R_{*}$ of the star on the basis of the photometric data.

The scalar quantity $\omega_{*}$ defined in Eq. (17) represents the emission frequency to be cross-correlated with the observational value $\omega_{\text {obs }}$ measured by the Gaia satellite. (The considerations made here about the single frequencies obviously apply to the whole spectrum, which is per se the observed object.) From Eqs. (15) and (17) it follows that, in terms of quantities defined in the rest frame of the local baricentric observer $\boldsymbol{u}$ at the event of emission, the reference data reads as

$\omega_{*}=-\gamma_{*}\left(v_{*}^{\alpha} k_{\alpha}+u^{\alpha} k_{\alpha}\right)_{*}$,

which, recalling Eqs. (8) and (18), gives

$\omega_{*}=-\gamma_{*}\left[v_{*}^{\alpha} k_{\alpha}+\left(1+M_{*} / R_{*}\right) k_{0}\right]_{*}$.

At the event of observation, the emitted spectrum with the single frequencies (20) will be compared with the observed spectrum whose single frequencies are obtained in a frame comoving with the satellite by the observer $\boldsymbol{u}_{\text {obs }}$ according to the formula

$\omega_{\mathrm{obs}}=-\left(u_{\mathrm{obs}}^{\alpha} k_{\alpha}\right)_{\mathrm{obs}}=-\gamma_{\mathrm{obs}}\left(v_{\mathrm{obs}}^{\alpha} k_{\alpha}+\frac{k_{0}}{\sqrt{-g_{00}}}\right)_{\mathrm{obs}}$,

where

$v_{\mathrm{obs}}^{\alpha}=-\frac{P(u)^{\alpha}{ }_{\beta} u_{\mathrm{obs}}^{\beta}}{u_{\mathrm{obs}}^{\rho} u_{\rho}}=\frac{u_{\mathrm{obs}}^{\alpha}}{\gamma_{\mathrm{obs}}}-u^{\alpha}$

and

$\gamma_{\mathrm{obs}}=-u_{\mathrm{obs}}^{\rho} u_{\rho}$ are the spatial three-velocity of the satellite and the corresponding Lorentz factor, respectively, relative to the local baricentric observer $\boldsymbol{u}$ located at the observation event.

Equations (20) and (21) are both expressed in terms of the spatial three-dimensional velocities of the star and of the satellite, relative to the respective local baricentric observers. While the components $v_{\mathrm{obs}}^{\alpha}$ refer to the motion of the satellite, which is expected to be known, the $v_{*}^{\alpha}$ represent the unknowns of our problem. Recalling Eq. (9), from Eq. (21) it follows that

$\omega_{\mathrm{obs}}=-\gamma_{\mathrm{obs}}\left(v_{\mathrm{obs}}^{\alpha} \ell_{\alpha}+\frac{k_{0}}{\sqrt{-g_{00}}}\right)_{\mathrm{obs}}$.

Since the frequencies $\omega_{*}$ and $\omega_{\text {obs }}$ represent two observables, their ratio

$\frac{\omega_{*}}{\omega_{\mathrm{obs}}}=\frac{\gamma_{*}\left[v_{*}^{\alpha} \ell_{\alpha}+\left(1+M_{*} / R_{*}\right) k_{0}\right]_{*}}{\gamma_{\mathrm{obs}}\left(v_{\mathrm{obs}}^{\alpha} \ell_{\alpha}+k_{0} / \sqrt{-g_{00}}\right)_{\mathrm{obs}}}$

is an experimentally measurable quantity. Using Eqs. (8)-(12) we obtain

$\frac{\omega_{*}}{\omega_{\mathrm{obs}}}=\frac{\left(k_{0}\right)_{*}}{\left(k_{0}\right)_{\mathrm{obs}}} \cdot \frac{\gamma_{*}\left[1-v_{*}^{\alpha}\left(\bar{\ell}_{\alpha}\right)_{*}\right]\left(1+M_{*} / R_{*}\right)}{\gamma_{\mathrm{obs}}\left(1-v_{\mathrm{obs}}^{\alpha} \bar{\ell}_{(0) \alpha}\right) / \sqrt{\left(-g_{00}\right)_{\mathrm{obs}}}}$,

where

$\overline{\boldsymbol{\ell}}_{(0)} \equiv(\overline{\boldsymbol{\ell}})_{\mathrm{obs}}$

is directly deducible from the observational data (de Felice \& Preti 2006).

The components $v_{*}^{\alpha}$ of the spatial three-velocity of the star with respect to the local baricentric observer can be decomposed in a radial plus a trasversal component relative to $\left(\bar{\ell}^{\alpha}\right)_{*}$, as follows:

$v_{*}^{\alpha}=-v_{*_{\mathrm{rad}}}\left(\bar{\ell}^{\alpha}\right)_{*}+v_{*_{\perp}}^{\alpha}$,

where

$v_{*_{\mathrm{rad}}} \equiv-v_{*}^{\rho}\left(\bar{\ell}_{\rho}\right)_{*}$,

provides the stellar radial velocity. The minus sign in definition (29) - which causes the minus sign in decomposition (28) - has been introduced for consistency with the usual conventions, according to which the radial velocity is positive when the star is moving away from the observer. (Recall that the direction of $\bar{\ell}$ is the incoming one, cf. Fig. 1 of de Felice et al. 2006.)

Indicating with $v_{*}$ the modulus of Eq. (28), and recalling that

$\gamma_{*}=\frac{1}{\sqrt{1-v_{*}^{2}}}$,

we see that Eq. (26) can be rewritten as

$$
\begin{aligned}
\frac{\omega_{*}}{\omega_{\mathrm{obs}}}= & \frac{\left(k_{0}\right)_{*}}{\left(k_{0}\right)_{\mathrm{obs}}} \frac{\sqrt{\left(-g_{00}\right)_{\mathrm{obs}}}}{\gamma_{\mathrm{obs}}\left(1-v_{\mathrm{obs}}^{\alpha} \bar{\ell}_{(0) \alpha}\right)} \\
& \times \frac{\left(1+v_{*_{\mathrm{rad}}}\right)\left(1+M_{*} / R_{*}\right)}{\sqrt{1-v_{*}^{2}}} .
\end{aligned}
$$

We now need to evaluate the ratio $\left(k_{0}\right)_{*} /\left(k_{0}\right)_{\text {obs }}$. The coordinate time direction is defined by the vector field $\xi^{\alpha}=\delta^{\alpha}{ }_{0}$, which is not a Killing field of spacetime (3). This implies that the scalar 
$k_{\alpha} \xi^{\alpha}=k_{0}$ is not a constant of motion along the null geodesic with tangent field $\boldsymbol{k}$; in fact, we have

$\frac{\mathrm{d}}{\mathrm{d} \lambda}\left(k_{\alpha} \xi^{\alpha}\right)=k^{\alpha} k^{\beta} \nabla_{\beta} \xi_{\alpha}$,

where $\nabla_{\beta}$ is the covariant derivative with respect to $x^{\beta}$ in the given metric, and $\lambda$ is a generic parameter along the null ray path. From the definition of covariant derivative, and recalling relations (4)-(7), we have

$\nabla_{\beta} \xi_{\alpha}=\partial_{\beta} \xi_{\alpha}-\frac{1}{2}\left(\partial_{\alpha} h_{0 \beta}+\partial_{\beta} h_{0 \alpha}-\partial_{0} h_{\alpha \beta}\right)+O(5)$.

Noting that, according to Eq. (9), the vector field $\boldsymbol{k}$ admits the decomposition

$k^{\alpha}=\ell^{\alpha}-\left(u^{\beta} k_{\beta}\right) u^{\alpha}$

and that $\bar{\ell}$, defined in Eqs. (12) and (13), is a unit vector, we can write Eq. (33) as

$$
\begin{aligned}
k^{\alpha} k^{\beta} \nabla_{\beta} \xi_{\alpha}= & \left(u_{\rho} k^{\rho}\right)^{2}\left(\bar{\ell}^{\alpha} \bar{\ell}^{\beta}+2 \bar{\ell}^{(\alpha} u^{\beta)}+u^{\alpha} u^{\beta}\right) \\
& \times\left(\partial_{\beta} h_{0 \alpha}-\partial_{(\alpha} h_{\beta) 0}+\frac{1}{2} \partial_{0} h_{\alpha \beta}\right)+O(5) \\
= & \left(u_{\rho} k^{\rho}\right)^{2}\left(\frac{1}{2} \bar{\ell}_{(0)}^{i} \bar{\ell}_{(0)}^{j} \partial_{0} h_{i j}+\frac{1}{2} \partial_{0} h_{00}\right)+O(5),
\end{aligned}
$$

where the last equivalence also follows from recalling that

$\bar{\ell}^{i}=\bar{\ell}_{(0)}^{i}+O(2)$

(cf. de Felice \& Clarke 1990). With a little algebra and observing that

$\frac{1}{\sqrt{-g_{00}}}=1+\frac{1}{2} h_{00}+O(4)$

we see that, at the relevant $O(3)$, Eq. (32) becomes

$\frac{\mathrm{d} k_{0}}{\mathrm{~d} \lambda}=\left(u_{\rho} k^{\rho}\right)^{2}\left(\frac{1}{2} \bar{\ell}_{(0)}^{i} \bar{\ell}_{(0)}^{j} \partial_{0} h_{i j}+\frac{1}{2} \partial_{0} h_{00}\right)$,

which since

$u_{\rho} k^{\rho}=\frac{k_{0}}{\sqrt{-g_{00}}}$,

and setting

$\mathrm{d} \sigma=\left(u_{\rho} k^{\rho}\right) \mathrm{d} \lambda$,

can also be written as

$\frac{\mathrm{d}}{\mathrm{d} \sigma} \ln k_{0}=\frac{1}{2}\left(\bar{\ell}_{(0)}^{i} \bar{\ell}_{(0)}^{j} \partial_{0} h_{i j}+\partial_{0} h_{00}\right)$,

thus leading to

$\frac{\left(k_{0}\right)_{\mathrm{obs}}}{\left(k_{0}\right)_{*}}=\exp \left[\frac{1}{2} \int_{\sigma_{*}}^{\sigma_{0}}\left(\bar{\ell}_{(0)}^{i} \bar{\ell}_{(0)}^{j} \partial_{0} h_{i j}+\partial_{0} h_{00}\right) \mathrm{d} \sigma\right]$

where $\sigma_{0} \equiv \sigma_{\text {obs }}$.

Now, using Eq. (42) and recalling Eq. (18), we see that Eq. (31) can be rewritten as

$$
\begin{aligned}
\frac{\omega_{*}}{\omega_{\mathrm{obs}}}= & \frac{\sqrt{\left(-g_{00}\right)_{\mathrm{obs}}}}{\gamma_{\mathrm{obs}}\left(1-v_{\mathrm{obs}}^{\alpha} \bar{\ell}_{(0) \alpha}\right)} \cdot \frac{\left(1+v_{*_{\mathrm{rad}}}\right)\left(1+M_{*} / R_{*}\right)}{\sqrt{1-v_{*}^{2}}} \\
& \times \exp \left[-\frac{1}{2} \int_{\sigma_{*}}^{\sigma_{0}}\left(\bar{\ell}_{(0)}^{i} \bar{\ell}_{(0)}^{j} \partial_{0} h_{i j}+\partial_{0} h_{00}\right) \mathrm{d} \sigma\right],
\end{aligned}
$$

which includes all the relevant contributions that must be taken into account for consistently dealing with the observational data from the new generation of astrometric satellites.

Equation (43) can actually be simplified further, since only the terms up to and including the $O(3)$ level have to be retained. Recalling Eqs. (18) and (37), and defining

$\chi \equiv-v_{\mathrm{obs}}^{\alpha} \bar{\ell}_{(0) \alpha}$,

using

$\gamma_{\mathrm{obs}}^{-1}=1-\frac{1}{2} v_{\mathrm{obs}}^{2}+O(4)$,

$(1+\chi)^{-1}=1-\chi+\chi^{2}-\chi^{3}+O(4)$,

$\exp \left[-\frac{1}{2} \int_{\sigma_{*}}^{\sigma_{0}}\left(\bar{\ell}_{(0)}^{i} \bar{\ell}_{(0)}^{j} \partial_{0} h_{i j}+\partial_{0} h_{00}\right) \mathrm{d} \sigma\right]=$
$1-\frac{1}{2} \int_{\sigma_{*}}^{\sigma_{0}}\left(\bar{\ell}_{(0)}^{i} \bar{\ell}_{(0)}^{j} \partial_{0} h_{i j}+\partial_{0} h_{00}\right) \mathrm{d} \sigma+O(4)$,

and introducing the shorthand

$$
\begin{aligned}
\zeta \equiv \frac{1}{2} & {\left[v_{\mathrm{obs}}^{2}+\left(h_{00}\right)_{\mathrm{obs}}\right.} \\
& \left.+\int_{\sigma_{*}}^{\sigma_{0}}\left(\bar{\ell}_{(0)}^{i} \bar{\ell}_{(0)}^{j} \partial_{0} h_{i j}+\partial_{0} h_{00}\right) \mathrm{d} \sigma-\frac{2 M_{*}}{R_{*}}\right],
\end{aligned}
$$

we finally obtain

$$
\begin{aligned}
\frac{\omega_{*}}{\omega_{\mathrm{obs}}}= & 1+\left(v_{*_{\mathrm{rad}}}-\chi\right)+\left(\frac{1}{2} v_{*}^{2}+\chi^{2}-\zeta-\chi v_{*_{\mathrm{rad}}}\right) \\
& +\left(v_{*_{\mathrm{rad}}}-\chi\right)\left(\frac{1}{2} v_{*}^{2}+\chi^{2}-\zeta\right)+O(4)
\end{aligned}
$$

where the terms in the righthand side have been explicitly grouped according to their progressive order of magnitude.

\section{Comparison with a previous formula}

In Lindegren \& Dravins (2003), a detailed review of the definition of "radial velocity" in astronomy is presented, with a critical revision of the equations usually employed to determine this velocity from the spectroscopic and astrometric measurements. In that paper, a formula for the Doppler shift is also given, with the aim of including all the corrections caused by the local kinematical and gravitational effects. This formula reads

$$
\begin{aligned}
\frac{\omega_{*}}{\omega_{\mathrm{obs}}}= & \left(1-\Phi_{\mathrm{obs}}-\frac{1}{2} v_{\mathrm{obs}}^{2}\right)\left(1+\boldsymbol{k}^{\prime} \cdot \boldsymbol{v}_{\mathrm{obs}}\right)^{-1} \\
& \times\left(1+\boldsymbol{k}^{\prime} \cdot \boldsymbol{v}_{*}\right)\left(1-\Phi_{*}-\frac{1}{2} v_{*}^{2}\right)^{-1},
\end{aligned}
$$

where use of the BCRS is implied. The Newtonian gravitational potentials are defined as $\Phi(\boldsymbol{r})=G \sum_{i} M_{i}\left|\boldsymbol{r}-\boldsymbol{r}_{i}\right|^{-1}, \Phi_{\mathrm{obs}}$ is the Newtonian gravitational potential at the observer location, $\Phi_{*}$ is the Newtonian gravitational potential of the star, $\boldsymbol{v}_{\text {obs }}$ and $\boldsymbol{v}_{*}$ are the three-dimensional Euclidean coordinate velocities of the observer and of the star, respectively, $\boldsymbol{k}^{\prime}$ is the three-dimensional Euclidean coordinate direction from the observer to the star, and the scalar products refer to three-dimensional Euclidean space.

Given the previous existence of Eq. (50), it is interesting and instructive to compare its $O(3)$ expansion with our Eq. (49). This 
comparison will reveal the importance of a consistently generalrelativistic analysis for determining a Doppler-shift formula adequate for the capabilities of the new generation of astrometric satellites and for the need of interpreting their spectroscopic observables in terms of the stellar radial velocities.

If we define

$\tilde{v}_{*_{\mathrm{rad}}} \equiv \boldsymbol{v}_{*} \cdot \boldsymbol{k}^{\prime}$

$\tilde{\chi} \equiv \boldsymbol{v}_{\mathrm{obs}} \cdot \boldsymbol{k}^{\prime}$

$\zeta_{\mathrm{obs}} \equiv \frac{1}{2}\left(v_{\mathrm{obs}}^{2}+2 \Phi_{\mathrm{obs}}\right)$,

hence rewrite Eq. (50) as

$$
\begin{aligned}
\frac{\omega_{*}}{\omega_{\mathrm{obs}}}= & \left(1-\zeta_{\mathrm{obs}}\right)(1+\tilde{\chi})^{-1} \\
& \times\left(1+\tilde{v}_{*_{\mathrm{rad}}}\right)\left(1-\Phi_{*}-\frac{1}{2} v_{*}^{2}\right)^{-1},
\end{aligned}
$$

recall expansion (46), and, finally, define

$\tilde{\zeta} \equiv \zeta_{\mathrm{obs}}-\Phi_{*}=\frac{1}{2}\left[v_{\mathrm{obs}}^{2}+2\left(\Phi_{\mathrm{obs}}-\Phi_{*}\right)\right]$,

we see that Eq. (50) power expands into

$$
\begin{aligned}
\frac{\omega_{*}}{\omega_{\mathrm{obs}}}= & 1+\left(\tilde{v}_{*_{\mathrm{rad}}}-\tilde{\chi}\right)+\left(\frac{1}{2} v_{*}^{2}+\tilde{\chi}^{2}-\tilde{\zeta}-\tilde{\chi} \tilde{v}_{*_{\mathrm{rad}}}\right) \\
& +\left(\tilde{v}_{*_{\mathrm{rad}}}-\tilde{\chi}\right)\left(\frac{1}{2} v_{*}^{2}+\tilde{\chi}^{2}-\tilde{\zeta}\right)+O(4) .
\end{aligned}
$$

Although formally analogous to Eq. (49), the above equation contains some substantial differences from it, and they mirror the differences existing between the "tilded" quantities (51) and (55), and the corresponding "untilded" ones (29) and (48). Noting that

$k^{\prime i}=-\bar{\ell}_{(0)}^{i}$,

we see that definitions (44) and (52) agree, namely

$\tilde{\chi}=\chi$,

but

$\tilde{v}_{*_{\mathrm{rad}}}=v_{*}^{i} k_{i}^{\prime}=-v_{*}^{\alpha} \bar{\ell}_{(0) \alpha} \neq-v_{*}^{\alpha}\left(\bar{\ell}_{\alpha}\right)_{*}=v_{*_{\mathrm{rad}}}$,

because of Eq. (36); however, because of this same equation we have

$\tilde{v}_{*_{\mathrm{rad}}}=v_{*_{\mathrm{rad}}}(1+O(2))$,

so we recognize that at first order Eqs. (56) and (43) coincide. Nevertheless, this coincidence is soon lost, already at second order: if we compare relation (55) with (48), we clearly see that the former completely misses the "history" of the photon (namely, the integral term appearing in the latter), which only results from a consistently general-relativistic analysis. Thus, although the velocity and potential terms in Eqs. (55) and (48) do correspond (note that $\left.\left(h_{00}\right)_{\mathrm{obs}}=2 \Phi_{\mathrm{obs}}\right)$, the absence of the "photon history" contribution in the former does represent a critical issue. This term does provide a $O(2)$ correction, so it is clear that its inclusion is mandatory for a consistent analysis of the satellite data, which are actually expected to reach the notably higher $O(3)$ precision. Moreover, we see that at $O(3)$ too Eq. (56) misses relevant corrections, which are provided by Eq. (49): not only the "photon history" integral, once again, but also the contribution arising from relations (59)-(60).

In conclusion, Eq. (50) is only correct to $O(1)$. It therefore cannot be consistently employed for any higher order analysis. For a consistent dealing with data from future astrometric satellites, recourse should be made to Eq. (49) instead.

\section{Separation of the radial velocity from the proper motion}

Wishing to determine the stellar velocity, we see that Eq. (49) alone does not allow this problem to be solved, because it contains two unknowns, namely the radial velocity $v_{*_{\text {rad }}}$ and the total velocity $v_{*}$ of the star, or equivalently, the radial velocity $v_{*_{\text {rad }}}$ and the proper motion $v_{*_{\perp}}$, according to Eq. (28).

An experimental method for separating the two velocity components, hence obtaining two independent equations for the two unknowns, is provided by the simultaneous observation of the incoming photons along two opposite directions, with the aid of a mirror to be included in the spectroscopic device, following a suggestion originally given by Ives \& Stilwell (1938) and also employed in Hasselkamp et al. (1979) for determining the special-relativistic transverse Doppler shift.

However, should such a direct spectroscopic measurement of the proper motion not be allowed by the satellite equipment, we could rely on the astrometric determination of the same, reducing Eq. (43) to an equation where the only unknown is the radial velocity $v_{*_{\text {rad }}}$, which can thus be determined in terms of both the astrometric and the spectroscopic data. In fact, it can be noted that the total velocity $v_{*}$ introduced so far - which is the instantaneous spatial three-velocity of the star with respect to the locally baricentric observer $\boldsymbol{u}$ at the emission event - actually coincides with the one determined in de Felice \& Preti (2006) via astrometric methods alone. This is because the local baricentric observer has no spatial velocity with respect to the BCRS, and the background metric for the stellar motion is the asymptotic form of Eq. (3); by the very definition of the instantaneous relative spatial velocity, these two total velocities do coincide, and the expression found for the latter can be correctly employed for the former as well.

\section{Explicit solution of the general-relativistic Doppler-shift formula}

The general-relativistic Doppler-shift formula (49) obtained above contains all the relativistic corrections up to $O(3)$ included, in agreement with the expected satellite astrometric capabilities. If we now write

$v_{*_{\mathrm{rad}}}=v_{*_{\mathrm{rad}(1)}}+v_{*_{\mathrm{rad}(2)}}+v_{*_{\mathrm{rad}(3)}}+O(4)$,
$v_{*_{\perp}}=v_{*_{\perp(1)}}+v_{*_{\perp(2)}}+v_{*_{\perp(3)}}+O(4)$,

and solve Eq. (49) in successive approximations, we find an explicit expression for the radial velocity in terms of the astrometric and spectroscopic data, namely

$$
\begin{aligned}
v_{*_{\mathrm{rad}}}= & \left(\frac{\omega_{*}}{\omega_{\mathrm{obs}}}-1\right)+\chi \\
& -\frac{1}{2}\left[\left(\frac{\omega_{*}}{\omega_{\mathrm{obs}}}-1\right)^{2}+\chi^{2}-2 \zeta+v_{*_{\perp(1)}}^{2}\right] \\
& -\left\{\left[\left(\frac{\omega_{*}}{\omega_{\mathrm{obs}}}-1\right)+\chi\right]\left(\frac{\omega_{*}}{\omega_{\mathrm{obs}}}-1\right) \chi+v_{*_{\perp(1)}} v_{*_{\perp(2)}}\right\} \\
& +O(4),
\end{aligned}
$$

which accounts for all the necessary first-, second-, and thirdorder corrections to Eq. (1) caused by the motion of the satellite, its position in spacetime, the photon history from the star to the satellite, and the surface gravity and the proper motion of the star itself. 


\section{Conclusions}

Using the Newtonian formula (1) to interpret the spectroscopic data is clearly unable to account for any relativistic effects. Working in the RAMOD framework, we have shown how the spectroscopic and astrometric data that will be provided by the new generation of satellites can be implemented with one another, thus leading to a general-relativistic Doppler formula Eq. (49) - which is exact up to and including the $O(3)$ terms, namely the order of magnitude consistent with the expected $\mu$ arcsec performance of the future astrometric satellite measurements. We also showed that a previously proposed Doppler-shift formula, Eq. (50), is definitely not adequate to this task, since it misses relevant relativistic corrections already at $O(2)$ and is therefore correct only at $O(1)$. Finally, by solving Eq. (49) we have been able to provide an explicit $O(3)$ expression - Eq. (63) - for the stellar radial velocity, in terms of the astrometric and spectroscopic data, where all the relevant relativistic contributions are now considered.

\section{References}

Bini, D., Crosta, M. T., \& de Felice, F. 2003, Class. Quantum Grav., 20, 4695 Crosta, M. T., \& Vecchiato, A. 2010, A\&A, 509, A37 de Bruijne, J., Perryman, M., Lindegren, L., et al. 2005, Gaia astrometric, photometric, and radial-velocity performance assessment methodologies to be used by the industrial system-teams, Technical Note Gaia-JdB-022, June 9

de Felice, F., \& Clarke, C. J. S. 1990, Relativity on Curved Manifolds (New York: Cambridge University Press)

de Felice, F., \& Preti, G. 2006, Class. Quantum Grav., 23, 5467

de Felice, F., \& Preti, G. 2008, Class. Quantum Grav., 25, 165015

de Felice, F., Lattanzi, M. G., Vecchiato, A., \& Bernacca, P. L. 1998, A\&A, 332, 1133

de Felice, F., Lattanzi, M. G., \& Vecchiato, A. 2001, A\&A, 373, 336

de Felice, F., Crosta, M. T., Vecchiato, A., Bucciarelli, B., \& Lattanzi, M. G. 2004, ApJ, 607, 580

de Felice, F., Vecchiato, A., Crosta, M. T., Bucciarelli, B., \& Lattanzi, M. G. 2006, ApJ, 653, 1552

Gullberg, D., \& Lindegren, L. 2002, A\&A, 390, 383

Hasselkamp, D., Mondry, E., \& Scharmann, A. 1979, Z. Phys. A, 289, 151

IAU 2000, Definition of Barycentric Celestial Reference System and Geocentric Celestial Reference System, IAU Resolution B1.3 adopted at the 24th General Assembly, Manchester, August

Ives, H. E., \& Stilwell, G. R. 1938, J. Opt. Soc. Am., 28, 215

Lindegren, L., \& Dravins, D. 2003, A\&A, 401, 1185

Preti, G., \& de Felice, F. 2010, A\&A, 513, A68

Turon, C., O'Flaherty, K. S., \& Perryman, M. A. C. 2005, The Threedimensional Universe with Gaia (The Netherlands: ESA Publication Division), SP-576 